\title{
EXTENSIONS OF WEAKLY SUPPLEMENTED MODULES
}

\author{
RAFAIL ALIZADE and ENGIN BÜYÜKAŞIK
}

\begin{abstract}
It is shown that weakly supplemented modules need not be closed under extension (i.e. if $U$ and $M / U$ are weakly supplemented then $M$ need not be weakly supplemented). We prove that, if $U$ has a weak supplement in $M$ then $M$ is weakly supplemented. For a commutative ring $R$, we prove that $R$ is semilocal if and only if every direct product of simple $R$-modules is weakly supplemented.
\end{abstract}

\section{Introduction}

Throughout, $R$ is a commutative ring with identity and $M$ is a unital left $R$ module. By $N \subseteq M$, we mean that $N$ is a submodule of $M$. A submodule $L \subseteq M$ is said to be essential in $M$, denoted as $L \unlhd M$, if $L \cap N \neq 0$ for every nonzero submodule $N \subseteq M$. A submodule $S$ of $M$ is called small (in $M$ ), denoted as $S \ll M$, if $M \neq S+L$ for every proper submodule $L$ of $M$. By $\operatorname{Rad} M$ we denote the sum of all small submodules of $M$ or, equivalently the intersection of all maximal submodules of $M$. A ring $R$ is said to be semilocal if $R / \operatorname{Rad} R$ is semisimple. By ([7] Proposition 20.2) $R$ is semilocal if and only if $R$ has only finitely many maximal ideals. A module $M$ is supplemented (see [12]), if every submodule $N$ of $M$ has a supplement, i.e. a submodule $K$ minimal with respect to $N+K=M . K$ is a supplement of $N$ in $M$ if and only if $N+K=M$ and $N \cap K \ll K$ (see [12]). If $N+K=M$ and $N \cap K \ll M$, then $K$ is called a weak supplement of $N$ (see, [14] and [8]). $M$ is a weakly supplemented module if every submodule of $M$ has a weak supplement. By $\Omega$ we denote the set of all maximal ideals of $R$. Let $R$ be a domain and $M$ be an $R$-module. The submodule $T(M)=\{m \in M \mid r m=0$ for some $0 \neq r \in R\}$ is called the torsion submodule of $M$, and if $M=T(M)$ then $M$ is called a torsion module.

Let $R$ be a Dedekind domain and $\mathfrak{p} \in \Omega$. The submodule $T_{\mathfrak{p}}(M)=\{m \in$ $M \mid \mathfrak{p}^{n} m=0$ for some $\left.n>0\right\}$ is called the $\mathfrak{p}$-primary part of $M$, and $T(M)=\bigoplus_{\mathfrak{p} \in \Omega} T_{\mathfrak{p}}(M)$ (see Proposition 10.6.9 in [3]). 
A class $\mathcal{M}$ of modules is said to be closed under extension if $U, M / U \in \mathscr{M}$ implies $M \in \mathscr{M}$. In this case we say that $M$ is an extension of $U$ by $M / U$.

Let $R$ be a noetherian local ring. Rudlof proved that an $R$-module $M$ is weakly supplemented if and only if it is an extension of a supplemented module by a supplemented module (see Theorem 3.1 in [10]). He also proved that over a noetherian ring every extension of a supplemented module by a supplemented module is weakly supplemented (see Proposition 3.6 in [10]). In general a weakly supplemented modules need not be an extension of a supplemented module by a supplemented module. For example the Z-module Q is weakly supplemented and Q does not contain any supplemented submodule (see [13], Theorem 3.1).

In this paper we show that the class of weakly supplemented modules need not be closed under extensions, that is if $U$ and $M / U$ are weakly supplemented for some submodule $U$ of $M$ then $M$ need not be weakly supplemented. But if $U$ has a weak supplement in $M$ we show that $M$ is weakly supplemented. We prove that a commutative ring $R$ is semilocal if and only if every direct product of simple $R$-modules is weakly supplemented. Let $R$ be a Dedekind domain. We obtain that an $R$-module $M$ is weakly supplemented if and and only if $T(M)$ and $M / T(M)$ are weakly supplemented and $T(M)$ has a weak supplement in $M$. If $M$ is a torsion $R$-module with $\operatorname{Rad} M \ll M$ then every submodule of $M$ is weakly supplemented.

\section{Extensions of weakly supplemented modules}

A submodule $N$ of a module $M$ is called closed in $M$ if $N \unlhd K$ for some $K \subseteq M$ implies $K=N$. A submodule $N$ of $M$ is called coclosed in $M$ if $N / K \ll M / K$ for some $K \subseteq M$ implies $K=N$.

Theorem 2.1. Let $0 \rightarrow L \rightarrow M \rightarrow N \rightarrow 0$ be a short exact sequence. If $L$ and $N$ are weakly supplemented and $L$ has a weak supplement in $M$ then $M$ is weakly supplemented.

If $L$ is coclosed in $M$ then the converse holds, that is if $M$ is weakly supplemented then $L$ and $N$ are weakly supplemented.

Proof. Without restriction of generality we will assume that $L \subseteq M$. Let $S$ be a weak supplement of $L$ in $M$ i.e. $L+S=M$ and $L \cap S \ll M$. Then we have,

$$
M /(L \cap S)=L /(L \cap S) \oplus S /(L \cap S)
$$

$L / L \cap S$ is weakly supplemented as a factor module of $L$. On the other hand, $S /(L \cap S) \cong M / L \cong N$ is weakly supplemented. Then $M /(L \cap S)$ is weakly supplemented as a sum of weakly supplemented modules (see [8] Proposition 2.5). Therefore $M$ is weakly supplemented by ([8], Proposition 2.2 (4)). 
Suppose that $L$ is coclosed. Then $L \cap S \ll L$ by ([5], Lemma 1.1) i.e. $L$ is a supplement of $S$ in $M$. Therefore $L$ is weakly supplemented by ([8], Proposition 2.2 (5)).

Proposition 2.2. Let $R$ be a semilocal ring (not necessarily commutative) and $M$ be an $R$-module. Suppose $U \subseteq M$ such that $M / U$ is finitely generated. If $U$ is weakly supplemented then $M$ is weakly supplemented.

Proof. Suppose $M / U$ is generated by

$$
m_{1}+U, m_{2}+U, \ldots, m_{n}+U .
$$

For the submodule $K=R m_{1}+R m_{2}+\cdots+R m_{n}$ we have $U+K=M$. Then $M$ is weakly supplemented by ([8], Proposition 2.5).

The following well known lemma is given for completeness.

Lemma 2.3. Let $M$ be a module and $U$ be a finitely generated submodule of $M$ contained in $\operatorname{Rad} M$. Then $U$ is small in $M$.

A module $M$ is said to be locally noetherian if every finitely generated submodule of $M$ is noetherian.

Proposition 2.4. Let $M$ be a locally noetherian module and $X \subseteq \operatorname{Rad} M$. Suppose $M / X$ is finitely generated. If $X$ and $M / X$ are weakly supplemented then $M$ is weakly supplemented.

Proof. Since $M / X$ finitely generated, $X+L=M$ for some finitely generated submodule $L$ of $M$. Then $X \cap L \subseteq X \subseteq \operatorname{Rad} M$ is finitely generated, because $L$ is finitely generated and $M$ is locally noetherian. So $X \cap L \ll M$. Thus $L$ is a weak supplement of $X$ in $M$. Therefore $M$ is weakly supplemented by Theorem 2.1.

We shall give an example in order to prove that the class of weakly supplemented modules need be closed under extensions. The following lemmas will be useful to present this example.

Lemma 2.5 ([1], Lemma 4.4). Let $R$ be a Dedekind domain. For an $R$ module $M$ the following are equivalent:

(1) $M$ is injective,

(2) $M$ is divisible,

(3) $M=P M$ for every maximal ideal $P$ of $R$,

(4) $M$ does not contain any maximal submodule. 
Note that if $M$ is divisible module over a Dedekind domain then $\operatorname{Rad} M=$ $M$. Hence if $N$ is a module with $\operatorname{Rad} N=0$ then $N$ does not contain divisible submodule.

Lemma 2.6. Let $R$ be a domain and $\mathfrak{p}$ a maximal ideal of $R$. Then for every $\mathfrak{p}$-primary $R$-module $M, M / \operatorname{Rad} M$ is semisimple.

Proof. $\operatorname{Rad} M=\bigcap_{\mathfrak{q} \in \Omega} \mathfrak{q} M$. We will show that $\mathfrak{q} M=M$ for every $\mathfrak{q} \in$ $\Omega \backslash\{\mathfrak{p}\}$. Let $x \in M$, then $\mathfrak{p}^{n} x=0$ for some $n \in \mathbf{N}$. Since $\mathfrak{p}^{n}+\mathfrak{q}=R$, we have $1=p+q$ for some $p \in \mathfrak{p}^{n}$ and $q \in \mathfrak{q}$. So we get $x=p x+q x=q x \in \mathfrak{q} M$, hence $M=\mathfrak{q} M$. Therefore $\operatorname{Rad} M=\bigcap_{\mathfrak{q} \in \Omega} \mathfrak{q} M=\mathfrak{p} M$. Then since $R / \mathfrak{p}$ is a field $M / \operatorname{Rad} M=M / \mathfrak{p} M$ is semisimple $R / \mathfrak{p}$-module, and so it is semisimple as an $R$-module.

Corollary 2.7. Let $R$ be a Dedekind domain and $M$ a torsion $R$-module, then $M / \operatorname{Rad} M$ is semisimple.

Proof. Since $R$ is a Dedekind domain and $M$ a torsion $R$-module, we have

$$
M=\bigoplus_{\mathfrak{p} \in \Omega} T_{\mathfrak{p}}(M)
$$

Then

$$
\begin{aligned}
M / \operatorname{Rad} M & =\left[\oplus_{\mathfrak{p} \in \Omega} T_{\mathfrak{p}}(M)\right] /\left[\bigoplus_{\mathfrak{p} \in \Omega} \operatorname{Rad} T_{\mathfrak{p}}(M)\right] \\
& \cong \bigoplus_{\mathfrak{p} \in \Omega}\left[T_{\mathfrak{p}}(M) / \operatorname{Rad} T_{\mathfrak{p}}(M)\right]
\end{aligned}
$$

is semisimple by Lemma 2.6, and by Theorem 9.6 in [2].

Lemma 2.8. Let $R$ be a Dedekind domain and $K$ be the field of quotients of $R$. Then ${ }_{R} K$ is weakly supplemented.

Proof. Since $R$ is a Dedekind domain and $K / R$ is a torsion $R$-module, we have $K / R \cong \bigoplus_{P \in \Omega} T_{P}(K / R)$ so $K / R$ is supplemented by Theorem 2.4 and Theorem 3.1 in [13]. Since $R$ is finitely generated and $\operatorname{Rad} K=K$ we have $R \ll K$. Therefore $K$ is weakly supplemented by Proposition 2.2 (4) in [8].

Lemma 2.9. Let $R$ be a Dedekind domain and $\left\{\mathfrak{p}_{i}\right\}_{i \in I}$ be an infinite collection of distinct maximal ideals of $R$. Let $M=\prod_{i \in I}\left(R / \mathfrak{p}_{i}\right)$ be the direct product of the simple $R$-modules $R / \mathfrak{p}_{i}$ and $T=T(M)$ be the torsion submodule of $M$. Then the following hold,

(1) $M / T$ is divisible, therefore $M / T \cong K^{(J)}$ for some index set $J$,

(2) $\operatorname{Rad} M=0$.

Proof. (1) Let $\mathfrak{p}$ be a maximal ideal of $R$. Then $\mathfrak{p}(M / T)=(\mathfrak{p} M+T) / T$. Now if $\mathfrak{p}$ is not one of the ideals $\left\{\mathfrak{p}_{i}\right\}_{i \in I}$ then $\mathfrak{p} M+T=M$ and so $\mathfrak{p}(M / T)=$ 
$M / T$. Suppose $\mathfrak{p} \in\left\{\mathfrak{p}_{i}\right\}_{i \in I}$, say $\mathfrak{p}=\mathfrak{p}_{j}$ for some $j \in I$, then $\mathfrak{p} M=M(\bar{j})$ where $M(\bar{j})$ consists of those elements of $M$ whose $j$ th coordinate is zero. Let $M(j)$ be the submodule of $M$ whose all coordinates except $j$ th are zero. Clearly $M(j) \subseteq T$. Then $M=M(\bar{j})+M(j) \subseteq \mathfrak{p} M+T$, so $\mathfrak{p} M+T=M$ and hence $\mathfrak{p}(M / T)=M / T$. Therefore by Lemma $2.5 M / T$ is divisible, and since it is torsion-free we have $M / T \cong K^{(J)}$.

(2) $M / M(\bar{j}) \cong R / \mathfrak{p}_{j}$ is a simple module, so $M(\bar{j})$ is a maximal submodule of $M$ for every $j \in I$. Then we get $\operatorname{Rad} M \subseteq \bigcap_{j \in I} M(\bar{j})=0$.

THEOREM 2.10. For a commutative ring $R$, the following are equivalent.

(1) $R$ is semilocal,

(2) Every direct product of simple R-modules is semisimple,

(3) Every direct product of simple R-modules is weakly supplemented.

Proof. (1) $\Rightarrow$ (2) Let $\mathfrak{p}_{1}, \mathfrak{p}_{2}, \ldots, \mathfrak{p}_{n}$ be the maximal ideals of $R$. Then $\operatorname{Rad} R=\mathfrak{p}_{1} \cap \mathfrak{p}_{2} \cap \cdots \cap \mathfrak{p}_{n}=\mathfrak{p}_{1} \cdot \mathfrak{p}_{2} \ldots \mathfrak{p}_{n}$. Let $M$ be a direct product of simple $R$-modules. Since every simple $R$-module is isomorphic to one of the simple modules $R / \mathfrak{p}_{j}, j=1, \ldots, n$, we have $\mathfrak{p}_{1} \cdot \mathfrak{p}_{2} \ldots \mathfrak{p}_{n} M=0$. So that $M$ is an $R / \operatorname{Rad} R$-module. By the hypothesis $R / \operatorname{Rad} R$ is semisimple, and so $M$ is a semisimple $R / \operatorname{Rad} R$-module. Therefore $M$ is a semisimple $R$-module.

(2) $\Rightarrow$ (3) Obvious.

(3) $\Rightarrow$ (1) Let $M=\prod_{\mathfrak{p} \in \Omega}(R / \mathfrak{p})$. From the proof of Lemma 2.9(2) we have $\operatorname{Rad}(M)=0$. Since $M$ is weakly supplemented, $M$ is semisimple by Corollary 2.3 in [8]. So that $M=\prod_{\mathfrak{p} \in \Omega}(R / \mathfrak{p}) \cong \bigoplus_{\mathfrak{p} \in \Omega} \bigoplus_{I_{\mathfrak{p}}}(R / \mathfrak{p})$ for some index sets $I_{\mathfrak{p}}$. In this case $(1+\mathfrak{p})_{\mathfrak{p} \in \Omega} \in M$ can have only finitely many nonzero components in the last decomposition. Therefore $\Omega$ is finite, i.e. $R$ has only finitely many maximal ideals. Hence $R$ is semilocal.

ExAmple 2.11. Let $R$ and $M$ be as in Lemma 2.9 and $T=\oplus_{i \in I}\left(R / \mathfrak{p}_{i}\right)$ be the torsion submodule of $M$. Note that $T$ is semisimple, so it is weakly supplemented. Let $N$ be a submodule of $M$ such that $N / T \cong K$. Then $N / T$ is weakly supplemented by Lemma 2.8 . Note that $\operatorname{Rad} N=0$ by Lemma 2.9 and $N$ is not semisimple because $N / T \cong K$ is not semisimple. Hence by Corollary 2.3 in [8], $N$ is not weakly supplemented.

Remark 2.12. In Theorem 2.1 the hypothesis that $L$ has a weak supplement can not be omitted. Let $T$ and $N$ be as in Example 2.11. Then $T$ has no weak supplement in $N$. Otherwise we would have $T+A=M$ and $T \cap A \ll N$ for some submodule $A$ of $N$. Since $\operatorname{Rad} N=0$ we have $T \cap A=0$. So the sum $T+A=M$ is a direct sum and $N / T \cong A$ is divisible, a contradiction.

The proof of the following lemma is standard. 
Lemma 2.13 (see [6], Exercise 6.34). Let $R$ be a domain and $M$ be an $R$-module. Then the torsion submodule $T(M)$ of $M$ is closed in $M$.

Note that over a Dedekind domain a submodule is closed if and only if it is coclosed (see [13], Satz 3.4).

Proposition 2.14. Let $R$ be a Dedekind domain and $M$ be an $R$-module. Then the following holds.

(1) If $M$ is weakly supplemented then $T(M)$ and $M / T(M)$ are weakly supplemented. If $T(M)$ has a weak supplement in $M$ then the converse holds.

(2) If $\operatorname{Rad} T(M) \ll M$ then $M$ is weakly supplemented if and only if $T(M)$ has a weak supplement in $M$ and $M / T(M)$ is weakly supplemented.

(3) Suppose $M$ is torsion. Then $M$ is weakly supplemented if $\operatorname{Rad} M$ is weakly supplemented and has a weak supplement in $M$.

(4) Suppose $M / \operatorname{Rad} M$ is finitely generated and $\operatorname{Rad} M \unlhd M$. Then $M$ is weakly supplemented if $\operatorname{Rad} M$ is weakly supplemented.

Proof. (1) Suppose $M$ is weakly supplemented. Then $T(M)$ is a weak supplement in $M$. Since $T(M)$ is also coclosed it is a supplement in $M$ by ([5], Lemma 1.1). Then $T(M)$ and $M / T(M)$ are weakly supplemented by Proposition 2.2(5) in [8].

If $T(M)$ has a weak supplement then $M$ is weakly supplemented by Theorem 2.1.

(2) $T(M) / \operatorname{Rad} T(M)$ is semisimple by Lemma 2.7 so it is weakly supplemented. Then $T(M)$ is weakly supplemented by Proposition 2.2(4) in [8]. Then the proof is clear by (1).

(3) By Lemma $2.7 M / \operatorname{Rad} M$ is semisimple. Then the proof is clear by Theorem 2.1.

(4) Suppose $M / \operatorname{Rad} M$ is generated by

$$
m_{1}+\operatorname{Rad} M, m_{2}+\operatorname{Rad} M, \ldots, m_{n}+\operatorname{Rad} M
$$

Then for the finitely generated submodule $K=R m_{1}+R m_{2}+\cdots+R m_{n}$ we have $\operatorname{Rad} M+K=M$ and $K \cap \operatorname{Rad} M$ is finitely generated as $K$ is finitely generated, so $K \cap \operatorname{Rad} M \ll M$ by Lemma 2.3 i.e. $K$ is a weak supplement of $\operatorname{Rad} M$ in $M$.

By ([2] Proposition 9.15) $\operatorname{Rad}(M / \operatorname{Rad} M)=0$, and since $\operatorname{Rad} M \unlhd M$, $M / \operatorname{Rad} M$ is torsion. Therefore $M / \operatorname{Rad} M$ is semisimple by Lemma 2.7. Hence $M$ is weakly supplemented by Theorem 2.1 .

A module $M$ is called coatomic if every proper submodule of $M$ is contained in a maximal submodule of $M$. Over a commutative noetherian ring every 
submodule of a coatomic module is coatomic (see Lemma 1.1 in [15]). Note that coatomic modules have small radicals.

Proposition 2.15. Let $R$ be a Dedekind domain and $M$ be a torsion $R$ module. If $\operatorname{Rad} M \ll M$ then every submodule of $M$ is weakly supplemented.

Proof. The module $M / \operatorname{Rad} M$ is semisimple by Lemma 2.7. Since $\operatorname{Rad} M \ll M$, every submodule of $M$ is contained in a maximal submodule i.e. $M$ is coatomic. Let $N$ be a submodule of $M$. Then $N$ is coatomic so $\operatorname{Rad} N \ll N$, and since $N$ is torsion, $N / \operatorname{Rad} N$ is semisimple. Hence $N$ is weakly supplemented by Proposition 2.2(4) in [8].

A domain $R$ is said to be one-dimensional if $R / I$ is artinian for every nonzero ideal $I$ of $R$. One-dimensional domains are proper generalizations of Dedekind domains.

Lemma 2.16. Let $R$ be a ring, $I \leqslant R$ and $M$ be an $R$-module. If I $M$ has a weak supplement $K$ in $M$, then $K$ is a weak supplement of $I^{n} M$ in $M$ for every $n \geqq 1$.

Proof. By hypothesis $I M+K=M$. Then we have $I^{2} M+I K=I M$, so $I^{2} M+I K+K=I M+K$ which gives $I^{2} M+K=M$. Continuing in this way we get:

$$
I^{n} M+K=M \text { and } I^{n} M \cap K \subseteq I M \cap K \ll M .
$$

This means that $K$ is a weak supplement of $I^{n} M$ in $M$.

Proposition 2.17. Let $R$ be a one-dimensional domain and $M$ be an $R$ module. Suppose that I is a nonzero ideal of $R$. If $I^{n} M$ is weakly supplemented and $I^{k} M$ has a weak supplement in $M$ for some $k \leqq n$, then $M$ is weakly supplemented.

Proof. Since $R$ is a domain $I^{n} \neq 0$. So $R / I^{n}$ is an artinian ring because $R$ is one-dimensional. Then $M / I^{n} M$ is a supplemented $R / I^{n}$-module by Theorem 24.25 in [7] and Theorem 4.41 in [9]. Hence $M / I^{n} M$ is a weakly supplemented $R$-module. By Lemma 2.16, $I^{n} M$ has a weak supplement in $M$. Therefore by Theorem 2.1, $M$ is weakly supplemented.

Corollary 2.18. Let $R$ be a one-dimensional domain and $M$ be an $R$ module. If $r M$ is weakly supplemented for some $0 \neq r \in R$ and has a weak supplement in $M$ then $M$ is weakly supplemented.

ACKNOWLEDGEMENTS. The authors would like to thank the referee for the valuable suggestions and comments. 


\section{REFERENCES}

1. Alizade, R., Bilhan, G., and Smith, P. F., Modules whose maximal submodules have supplements, Comm. Algebra 29:6 (2001), 2389-2405.

2. Anderson, F. W., and Fuller, K. R., Rings and Categories of Modules, Springer, New York, 1992.

3. Cohn, P. M., Basic Algebra: Groups, Rings and Fields, Springer, London, 2002.

4. Kaplansky, I., Infinite Abelian Groups, Ann Arbor, Michigan: Michigan University Press, 1965.

5. Keskin, D., On lifting modules, Comm. Algebra $28: 7$ (2000), 3427-3440.

6. Lam, T. Y., Lectures on Modules and Rings, Springer, New York, 1999.

7. Lam, T. Y., A first course in Noncommutative Rings, Springer, New York, 1999.

8. Lomp, C., On semilocal modules and rings, Comm. Algebra 27:4 (1999), 1921-1935.

9. Mohamed, S. H., and Müller, B. J., Continuous and Discrete Modules, Cambridge University Press 1990.

10. Rudlof, P., On the structure of couniform and complemented modules, J. Pure Appl. Alg. 74 (1991), 281-305.

11. Santa-Clara, C., and Smith, P. F., Direct product of simple modules over Dedekind domains, Arch. Math. (Basel) 82 (2004), 8-12.

12. Wisbauer, R., Foundations of Modules and Rings, Gordon and Breach, 1991.

13. Zöschinger, H., Komplementierte Moduln über Dedekindringen, J. Algebra. 29 (1974), 42-56.

14. Zöschinger, H., Invarianten wesentlicher Überdeckungen, Math. Ann. 237 (1978), 193-202.

15. Zöschinger, H., Koatomare Moduln, Math. Z. 170 (1980), 221-232.

DEPARTMENT OF MATHEMATICS

IZMIR INSTITUTE OF TECHNOLOGY

GÜLBAHÇEKÖYÜ

35430 URLA IZMIR

TURKEY

E-mail: rafailalizade@iyte.edu.tr, enginbuyukasik@iyte.edu.tr 